\title{
Adjustment of $k-\omega$ SST turbulence model for an improved prediction of stalls on wind turbine blades
}

\author{
Tawit Chitsomboon ${ }^{*}$, Chalothorn Thamthae \\ School of Mechanical Engineering, Institute of Engineering, \\ Suranaree University of Technology, Nakornratchasima, Thailand \\ * Corresponding author. Tel: +66 044 224414, Fax: +66 044 224413, E-mail: tabon@sut.ac.th
}

\begin{abstract}
The eddy viscosity in the buffer zones of the turbulent boundary layers was limited to investigate its effects on the points of incipient separation for flows over wind turbine airfoils. The $k-\omega$ SST turbulence model was used as the base model within the framework of a finite volume CFD scheme. Flows over two different wind turbine airfoils were computed and compared with experimental results. Much improvements in the lift and drag coefficients in the stall regions were observed when compared with the results of the original turbulence model.
\end{abstract}

Keywords: Turbulence model, $k-\omega$ SST turbulence model, Airfoil stall prediction, Stall in wind turbine

\section{Introduction}

Practical flows over wind turbine blades at large angles of attack are very complicated, thus difficult to predict accurately by numerical methods. The difficulties stem from the fact that the boundary layers separate from the blade's suction surfaces due to adverse pressure gradients and its interaction with turbulence. Several investigations have been conducted by various researchers to compute these separated flows; the results have been hitherto only partially successful. Some investigations have over-predicted the lift coefficients [1-3] while some have under-predicted them [4-6]. Moreover, all computations missed the characteristic dips and rises of the lift coefficients in the regions right after the onsets of stalls [1-8]. The reasons for the inaccurate numerical predictions were invariably attributable to the inaccuracies incurred by the turbulence models used in the computations.

Early computations employed the $k-\varepsilon$ turbulence model [9] but later on the $k-\omega$ turbulence model [10] became more acceptable; at present the $k-\omega$ SST model [11-12] seems to be more preferable among wind turbine researchers. This model utilizes both the $k-\varepsilon$ and the $k-\omega$ models under a strategy of a blending function. Like it predecessors this model employs the fully turbulent flow condition as its basic assumption, i.e. the flow devoid of a transition region. The performances of this model in the predictions of separated flows over airfoils were only partially satisfactory since substantial inaccuracies of the lift and drag coefficients predictions were still prevailed in the stall regimes [1-8].

In particular, the $k-\omega$ SST turbulence model had been demonstrated to over-predict the lift coefficients beyond the stall points [1,5,6,8 ]. It is believed by the authors that these overpredictions were due to the fact that the turbulence levels (hence, turbulent eddy viscosities) in the boundary layers were too high, thus enhancing a momentum transfers to the near wall regions which helped the boundary layer to push through the adverse pressure gradient regions more easily than otherwise. The resulting delayed separation then caused the low pressure on the suction side to spread over more area than normal which thus increased the lift. The turbulence levels that were too high, in turn, were caused by the fully turbulent assumptions that were employed in the turbulence model. 
To this end, it should be noted that the buffer zone which bridges the laminar sub-layer and the log-law region in a boundary layer is very important to the physic of turbulence in the boundary layer. This zone is very difficult to model numerically. A small 'model error' in this relatively thin region could induce a large overall error.

The objective of this study was to improve the accuracy of the numerical prediction of separated flows on airfoils by an adjustment of the turbulence intensity in the buffer zones of turbulent boundary layers. The $k-\omega$ SST turbulence model $[11,12]$ will be used as the basis for this investigation.

\section{Methodology}

In order to limit the eddy viscosities in the regions from the buffer zones to the log-law zone a damping function $f_{S S T}$ is proposed. The limited eddy viscosity for the $k-\omega$ SST model thus becomes,

$$
\mu_{t}=f_{S S T} \min \left[\frac{\rho k}{\omega} ; \frac{a_{1} \rho k}{S F_{2}}\right]
$$

The damping function $f_{\text {SST }}$ was defined as a function of $y^{+}$(a scaled normal distance from the wall),

$$
\begin{array}{ll}
f_{\text {SST }}=f(<1.0) & ; a \leq y^{+} \leq b \\
f_{S S T}=1 & ; y^{+}<a, y^{+}>b
\end{array}
$$

For simplicity, the damping function was initially defined as a step function as shown in Figure 1 (solid line). Later, it was modified to be a continuous function. The constant $a$ was set in the range 5-30 and the constant $b$ was set in the range 50-300 for a parametric study. The values selected correspond to the edges of the buffer zone and the log law zone of a turbulent boundary layer.

The CFD code used in this study was the FLUENT Code [13]. The computational mesh was an O-type extending approximately to 35 chord lengths away from the airfoil and 250 points were employed along airfoil surfaces. The first cell at any surface point was sized such that $y^{+} \leq 2$ whereas grids away from it were expanded at a rate less than $20 \%$. This grid system was found to be suitable for a RANS simulation of turbulent boundary layer flows $[14,15]$. 


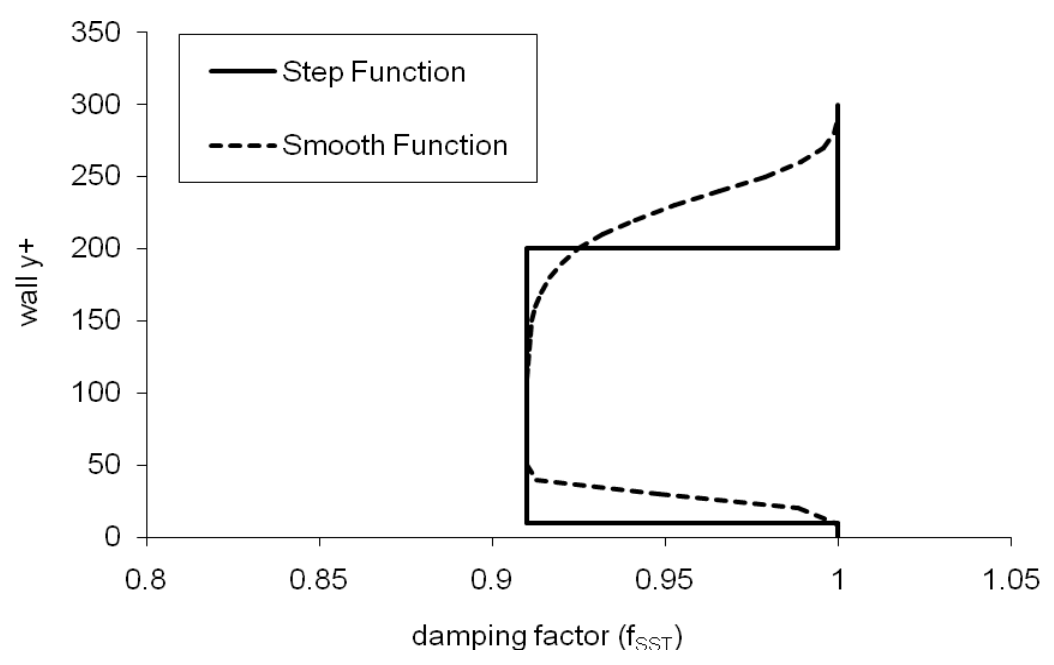

Fig. 1 Example of the step damping function and the hyperbolic (smooth) damping function.

Computations of flows over NREL's S809 airfoil [16] and NACA 63-215 [17] were conducted. An initially parametric study was performed to find out as to how sensitive a boundary layer would respond to changes in the values of $a, b$ and $f$, using the step function. For this purpose, only some values of angles of attack, namely: $9^{\circ}, 11^{\circ}, 15^{\circ}$ and $20^{\circ}$ were selected; these are critical points on the lift curve of the S809 airfoil.

\section{Results}

Figure 2 compares the various computed results with the experimental data at angles of attack $9^{\circ}$ and $11^{\circ}$. It can be seen that the lifts are quite sensitive to small changes in the values of $a$, $b$ and $f$. Actually, several numerical experiments had been performed to weed out the unreasonable ones; only the more meaningful results are demonstrated here.

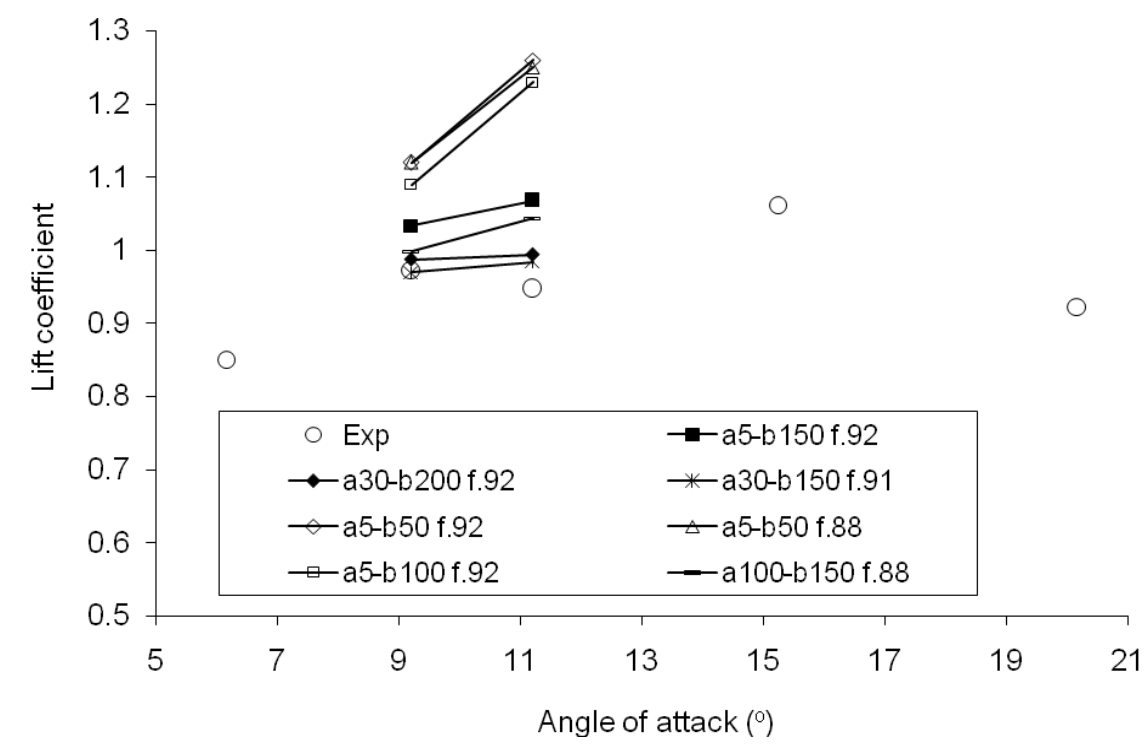

Fig.2 Effects of $a, b$ and $f$ in the step damping function on the lift coefficients.

After getting the feel for the sensitivity of the response, more numerical experiments were performed; the results of which are shown in Fig. 3 wherein it is observed that the characteristic dip and rise of the lift distributions are captured. The value of $f a$ and $b$ in the 
ranges $0.90-0.91,5-10$ and $100-200$, respectively, were found to be acceptable. Note that the range of acceptable $f$ is very narrow.

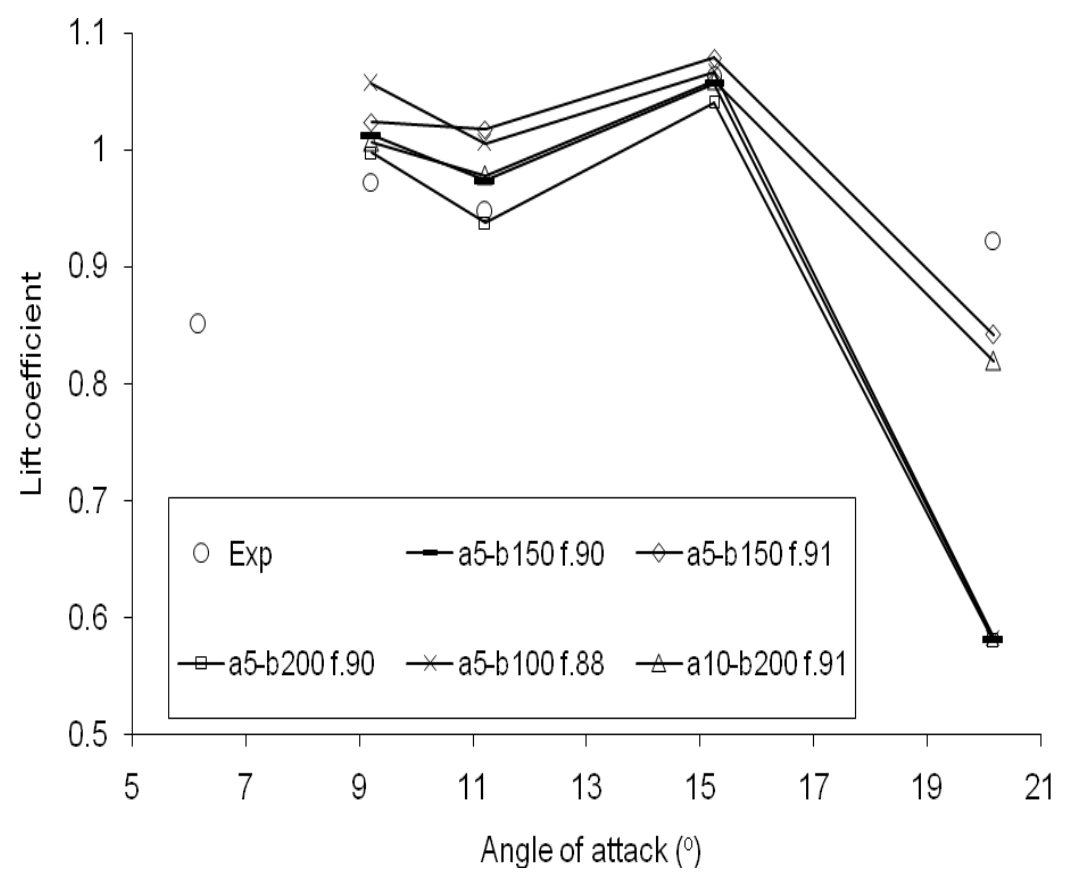

Fig. 3 Effects of $a, b$ and $f$ in the step damping function on lift at high angles of attack.

From the knowledge and experience gained in the parametric study of the step damping function, a continuous function is now proposed as,

$$
f_{S S T}=0.1+\left\{1-0.1 \tanh \left[\left(0.03 y^{+}\right)^{4}\right]\right\} \times\left\{0.9+0.1 \tanh \left[\left(0.005 y^{+}\right)^{8}\right]\right\}
$$

This damping function is the product of two hyperbolic functions; it was designed (by trial and error) for a rapid rise in the buffer zone and a gradual decrease in the log-law zone, as shown in Fig. 1 (dash line.)

Figure 4 compares the experimental results for lift on the S809 airfoil [16] to three computations, namely: 1) the present model by using the continuous damping function (denoted as SST+), 2) the 2-equation $k-\omega$ SST model (denoted as SST) and 3) the 4equation $k-\omega$ SST turbulence model with transition [8] (denoted as SST-T.) This last turbulence model is an enhanced version of its 2-equation counterpart wherein a model for transition to turbulence is also incorporated. It is clearly seen that, in the stall region, the SST+ results compare much more favorably to the experiment than those of the other two models. The improvements are not only in a quantitative manner but also in a qualitatively manner, notably the dip and rise of the distribution right after the incipient point of stall at about 10 degree angle of attack. Drag coefficient plot, shown in Fig. 5, also indicates that SST+ results are superior to other models. 


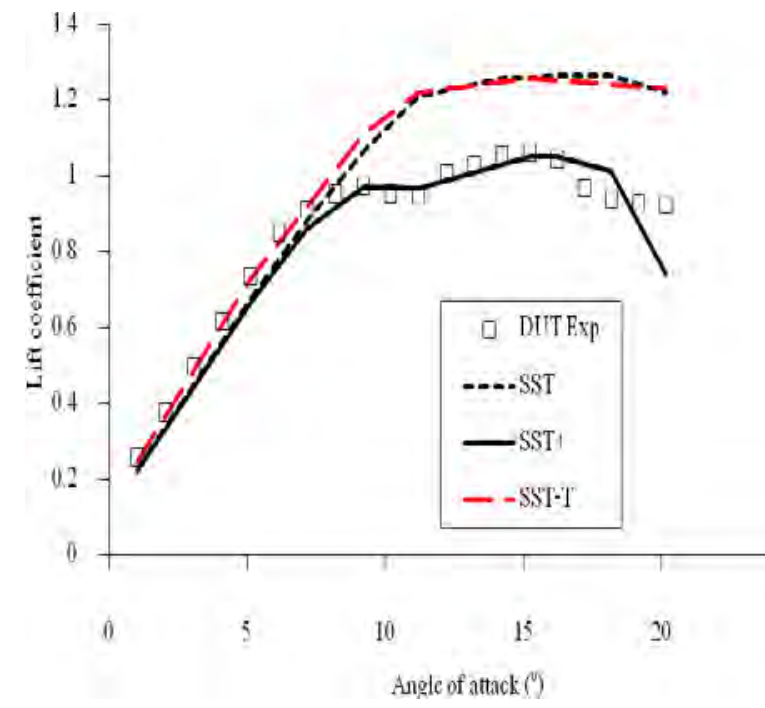

Fig. 4 Lift predictions on 5809 airfoil using present and original turbulence models

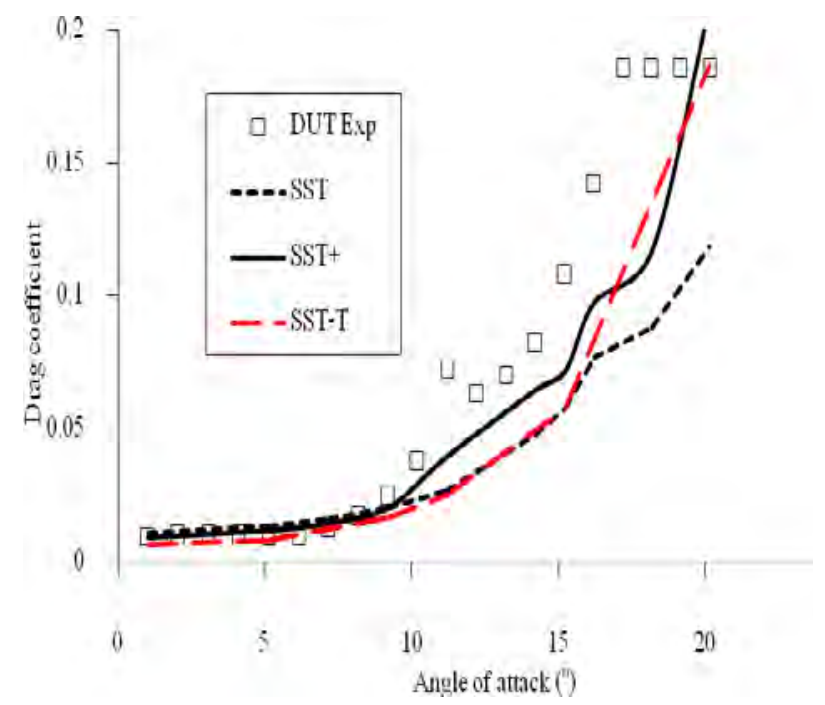

Fig. 5 Drag predictions on S809 airfoil using present and original turbulence models.

It is evident that in the low angle of attack region where the flow is still attached, SST-T gives best results but from the stall point onward SST-T exhibits over-predictions much in the same trend as SST. This perhaps is due to the fact that when flows separate on the suction side there is no transition regions left to give an advantage point to SST-T.

The predicted pressure distributions on the S809 airfoil surfaces at $14^{\circ}$ and $20^{\circ}$ angles of attack are shown in Figs. 6 and 7, respectively. The figures confirm that SST+ gives better overall agreements to the experiment than SST and SST-T. Specifically, the points of separation predicted by SST+ agree better with the experimental results; this is the main reason why it can predict aft-stall lift coefficients more accurately.

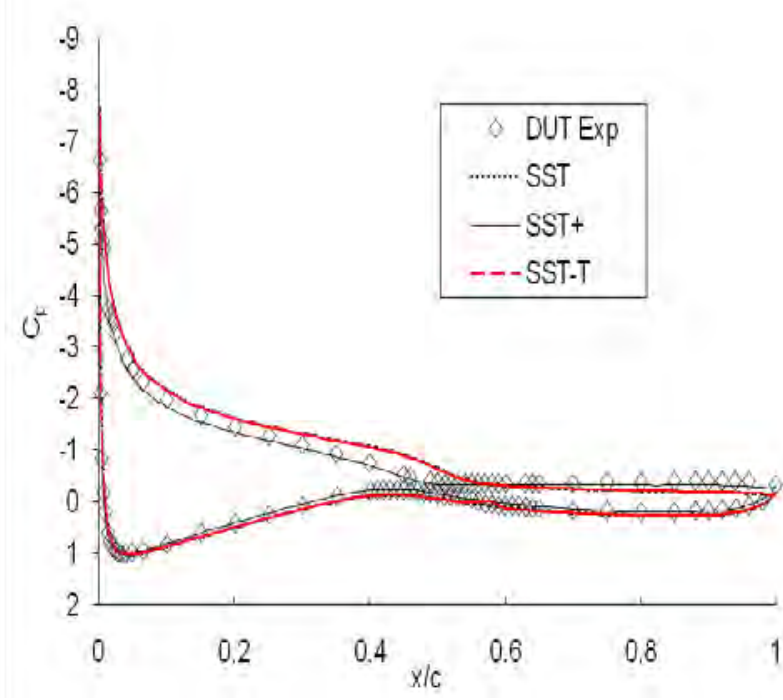

Fig.6 Comparison of pressure distributions on S809 airfoil at $14^{\circ}$ angle of attack

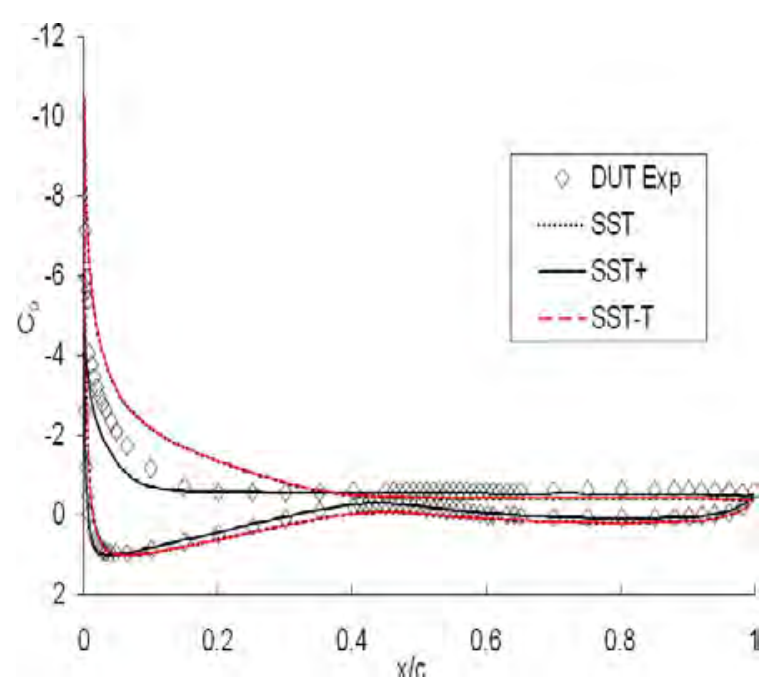

Fig.7 Comparisons of pressure distributions on S809 airfoil at $20^{\circ}$ angle of attack

The incipient of separations can be noticed as the point where the pressure distributions on the suction side are leveled off. Again, SST and SST-T give almost identical results. 
To verify that the improved results obtained by the adjustment made to the buffer zone was not specific to an airfoil, flows over another airfoil, the NACA 63-215 [17] were solved under similar conditions. The results are shown in Figs. 8 and 9. Again, SST+ gives much better results than the original models, both quantitatively and qualitatively.

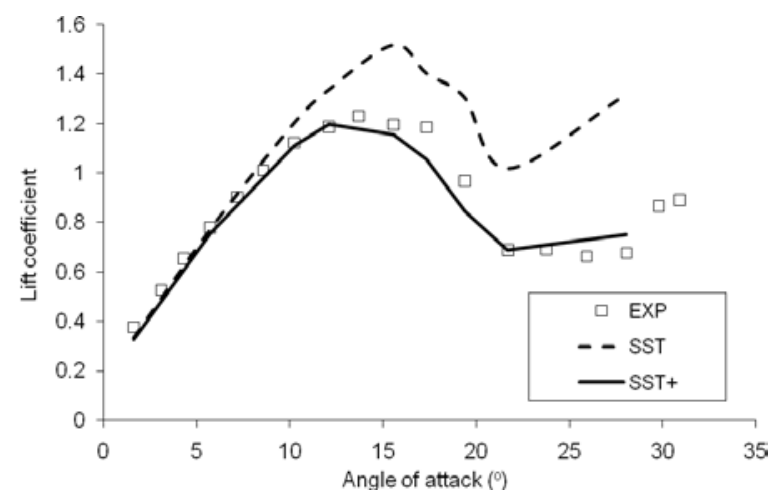

Fig.8 Comparison of lift between modified and original turbulence model.

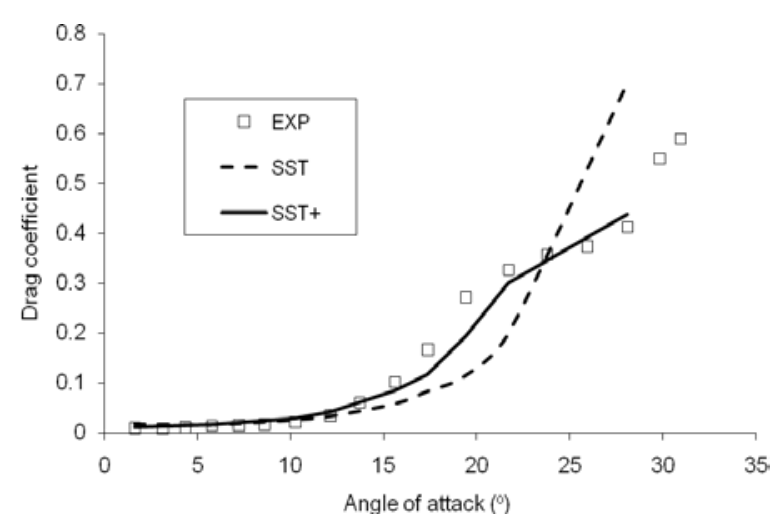

Fig.9 Comparison of drag between modified and original turbulence model.

\section{Discussion and Conclusions}

It could perhaps be reasoned that the improvements in lift and drag predictions over airfoils after stall were caused by the eddy viscosities in the buffer zones being reduced to about the right levels so as to compensate for the overall collective effects of the transition regions upstream that were neglected by the fully-developed turbulence model. In addition, compensations were also automatically provided for the local phenomena occurring within the buffer zones.

It has been evident that the behavior of a turbulent boundary layer over an airfoil is very sensitive to events in the buffer zone. A small change in the levels of turbulence intensity in the buffer zone could produce a dramatic change in the overall response of the separated flows on airfoils resulting in large changes on lift and drag.

By limiting the turbulence intensities (eddy viscosities) in the narrow buffer zones of turbulent boundary layers by about $10 \%$ together with the use of the $k-\omega$ SST turbulence model, this investigation has demonstrated that predictions of lifts and drags (after stall) on two independent airfoils were much improved. The improvements were evidently due to the reductions in the delay of incipient separation points which in turn were caused by the boundary layer being less energetic since the eddy viscosities (hence momentum transfers) were limited.

\section{References}

[1] P. Catalano and M. Amato, An evaluation of RANS turbulence modeling for aerodynamics, Aerospace Science and Technology 7, 2003, pp. 493-509.

[2] D.J. Mavriplis, Three-dimensional high-lift analysis using a parallel unstructured multigrid solver. AIAA Paper 98-2619, June 1998.

[ 3] C.L. Rumsey, T.B. Gatski, Recent turbulence model advances applied to multi element airfoil computations, J Aircraft 38(5), 2001, pp. 904-10 
[ 4] Wolfe, W.P. and Ochs, S.S. "CFD Calculations of S809 Aerodynamic Characteristics," AIAA-97-0973, Proceeding 35th AIAA Aerospace Sciences Meeting and Exhibit, Reno 1997

[ 5] Bertagnolio, F., Sørensen, N.N., and Johansen, J. (2006, December). Status for the TwoDimensional Navier-Stokes Solver EllipSys2D. Risø-R-1282(EN), Risø National Laboratory

[ 6] Chow, R. and van Dam, C.P., Computational Investigations of Deploying Load Control Microtabs on a Wind Turbine Airfoil, AIAA-2007-1018

[7] Rumsey, C.L., Ying, S.X., (2002). Prediction of high lift: review of present CFD capability. Progress in Aerospace Sciences 38: pp. 145-180

[ 8] Langtry, R.B., Gola, J., and Menter, F.R. (2006). Predicting 2D airfoil and 3D wind turbine rotor performance using a transition model for general CFD codes. 44th AIAA Aerospace Sciences Meeting and Exhibit, Reno 2006, AIAA 2006-0395

[9] Jones, W. P., and Launder B. E., The prediction of Laminarization with a TwoEquation Model of Turbulence, International Journal of Heat and Mass Transfer, Vol. 15, 1972, pp. 301-314

[ 10] Wilcox, D. C., (1993).Turbulence Modeling for CFD. DCW Industries, Inc., 5354 Palm Drive, La Cafiada, Calif.

[11] Menter, F.R. (1993). Zonal two equation $k-\omega$ turbulence models for aerodynamic flows. AIAA Paper 93-2906

[12] Menter, F.R. (1994, Nov). Two-equation eddy viscosity turbulence models for engineering applications. AIAA J 32:1299-1310.

[13] Fluent 6.3 User manual

[14] Franck Bertagnolio, Niels S_rensen and Jeppe Johansen, Status for the TwoDimensional Navier-Stokes Solver EllipSys2D, Risø-R-1282(EN)

[15] Cummings, R.M., Forsythe, J.R., Morton, S.A., and Squires, K.D., (2003). Computational challenges in high angle of attack flow prediction. Progress in Aerospace Sciences, 39:369384. doi:10.1016/S0376-0421(03)00041-1

[16] Somers, D.M., (1997). Design and experimental results for the S809 airfoil. Airfoils Inc., State College, PA. NREL/SR-440-6918

[17] Bertagnolio F., Sørensen N N., Johansen J., and Fuglsang P. (2001). Wind Turbine Airfoil Catalog, Risø-R-1280(EN), Risø National Laboratory, August 2001

\section{Acknowledgements}

This research was supported by the Royal Golden Jubilee Ph.D. program of the Thailand Research Fund. The authors are thankful to Prof. J.N. Sørensen of the Technological University of Denmark for his collaboration through the RGJ Ph.D. program. 